\title{
Optical validation and characterization of Planck PSZ2 sources at the Canary Islands observatories
}

\section{Second year of LP15 observations}

\author{
A. Aguado-Barahona ${ }^{1,2}$, R. Barrena ${ }^{1,2}$, A. Streblyanska ${ }^{1,2}$, A. Ferragamo ${ }^{1,2}$, J. A. Rubiño-Martín ${ }^{1,2}$, \\ D. Tramonte ${ }^{3,1,2}$, and H. Lietzen ${ }^{4}$
}

1 Instituto de Astrofísica de Canarias, C/Vía Láctea s/n, 38205 La Laguna, Tenerife, Spain
e-mail: aaguado@iac.es, alejandro. aguado. barahona@gmail. com
2 Universidad de La Laguna, Departamento de Astrofísica, 38206 La Laguna, Tenerife, Spain
3 University of KwaZulu-Natal, Westville Campus, Private Bag X54001, Durban 4000, South Africa
4 Tartu Observatory, University of Tartu, Observatooriumi 1, 61602 Tõravere, Estonia

Received 6 June 2019 / Accepted 9 September 2019

\begin{abstract}
Context. The second legacy catalog of Planck Sunyaev-Zeldovich (SZ) sources, hereafter PSZ2, provides the largest galaxy cluster sample selected by means of the SZ signature of the clusters in a full sky survey. In order to fully characterize this PSZ2 sample for cosmological studies, all the members should be validated and the physical properties of the clusters, including mass and redshift, should be derived. However, at the time of its publication, roughly $21 \%$ of the 1653 PSZ2 members had no known counterpart at other wavelengths.

Aims. Here, we present the second and last year of observations of our optical follow-up program 128-MULTIPLE-16/15B (hereafter LP15), which has been developed with the aim of validating all the unidentified PSZ2 sources in the northern sky with declinations higher than $-15^{\circ}$ that have no correspondence in the first Planck catalog PSZ1. The description of the program and the first year of observations have been presented previously.

Methods. The LP15 program was awarded 44 observing nights that were spread over two years with the Isaac Newton Telescope (INT), the Telescopio Nazionale Galileo (TNG), and the Gran Telescopio Canarias (GTC), all at Roque de los Muchachos Observatory (La Palma). Following the same method as described previously, we performed deep optical imaging for more than 200 sources with the INT and spectroscopy for almost 100 sources with the TNG and GTC at the end of the LP15 program. We adopted robust confirmation criteria based on velocity dispersion and richness estimates for the final classification of the new galaxy clusters as the optical counterparts of the PSZ2 detections.

Results. Here, we present the observations of the second year of LP15, as well as the final results of the program. The full LP15 sample comprises 190 previously unidentified PSZ2 sources. Of these, 106 objects were studied before, while the remaining sample (except for 6 candidates) has been completed in the second year and is discussed here. In addition to the LP15 sample, we here study 42 additional PSZ2 objects that were originally validated as real clusters because they matched a WISE or PSZ1 counterpart, but they had no measured spectroscopic redshift. In total, we confirm the optical counterparts for 81 PSZ2 sources after the full LP15 program, 55 of them with new spectroscopic information. Forty of these 81 clusters are presented in this paper. After the LP15 observational program the purity of the PSZ2 catalog has increased from $76.7 \%$ originally to $86.2 \%$. In addition, we study the possible reasons for false detection, and we report a clear correlation between the number of unconfirmed sources and galactic thermal dust emission.
\end{abstract}

Key words. galaxies: clusters: general - large-scale structure of Universe - catalogs

\section{Introduction}

Galaxy clusters (GCs) are a very powerful tool for testing the cosmological model (Allen et al. 2011). They have been used for cosmology studies since Zwicky's discovery of dark matter in the Coma cluster (Zwicky 1933), and based on the recent allsky surveys that appeared in the past decade, they have become studied significantly more frequently and in greater depth. In particular, the Planck ${ }^{1}$ satellite (Planck Collaboration I 2014) for the

\footnotetext{
1 Planck http://www . esa.int/Planck is a project of the European Space Agency (ESA) with instruments provided by two scientific consortia funded by ESA member states and led by Principal Investigators from France and Italy, telescope reflectors provided through a collaboration between ESA and a scientific consortium led and funded by Denmark, and additional contributions from NASA (USA).
}

first time provided the possibility for detecting GCs in a full-sky survey by means of the Sunyaev-Zeldovich (SZ) effect (Sunyaev \& Zeldovich 1972), which is the spectral distortion of the cosmic microwave background (CMB). As CMB photons pass through a GC, they can interact with the hot intracluster medium (ICM) electrons through inverse Compton scattering. As a result of this interaction, the $\mathrm{CMB}$ photons gain energy and the overall $\mathrm{CMB}$ spectrum is shifted toward higher frequencies, producing a characteristic spectral dependence that can be used to detect them.

In order to use the GCs surveys to constrain cosmological parameters (Planck Collaboration VI 2018; Planck Collaboration XXIV 2016), it is important to obtain unbiased measurements of the cluster mass and redshift. In particular, because the surface brightness of the SZ effect does not depend on redshift, follow-up campaigns at other wavelengths are needed in order to 
complement the SZ information. A detailed characterization of the Planck SZ survey cluster sample is needed to improve cosmological constraints from the Planck survey alone, and also to obtain constraints from future large galaxy cluster surveys, such as the Spectrum-Roentgen-Gamma/extended ROentgen Survey with an Imaging Telescope Array (SRG/eROSITA) cluster survey (Predehl et al. 2010), whose spacecraft was successfully launched on July 132019 and first light for the SRG/ eROSITA telescope is planned to be obtained very soon.

The PSZ2 catalog (Planck Collaboration XXVII 2016) is the second Planck catalog of SZ sources derived from the full 29 months of mission data. This catalog is based on the results from three cluster detection codes (MMF1, MMF3, and PwS) described in detail in Planck Collaboration XXIX (2014) and Planck Collaboration XXVII (2016). The PSZ2 was validated using external X-ray, optical, SZ, and near-IR data and at the time of its publication contained 1653 detections of which 1203 were confirmed as actual galaxy clusters, 1094 of them including redshifts. The PSZ2 catalog contains all objects found by at least one of the three detection algorithms with a signal-to-noise ratio $S / N \geq 4.5$ for the $\mathrm{SZ}$ detection.

A first validation process was performed in Planck Collaboration XXVII (2016). It began using a cross match with the PSZ1, continuing the search for possible counterparts in the Meta-Catalog of the compiled properties of X-ray detected Clusters of galaxies (MCXC) catalog (Piffaretti et al. 2011), which is based on the ROSAT All Sky Survey (RASS; Voges et al. 1999, 2000) and on the serendipitous ROSAT and Einstein cluster catalogs, in the Sloan Digital Sky Survey (SDSS; York et al. 2000), in the redMaPPer catalog (Rykoff et al. 2014), in the NED ${ }^{2}$, in similar follow-ups (Planck Collaboration Int. XXVI 2015; Planck Collaboration Int. XXXVI 2016) as we present here, in the AllWISE mid-infrared source catalog (Cutri et al. 2013), and in SZ catalogs such as those obtained with data from the South Pole Telescope (SPT; Bleem et al. 2015), the Atacama Cosmology Telescope (ACT; Hasselfield et al. 2013) and by direct follow-up with the Arcminute Micro-kelvin Interferometre (AMI; Perrott et al. 2015).

This paper is the second in a series of papers in which optical characterization of SZ sources was performed using the Canary Islands Observatories. Planck Collaboration Int. XXXVI (2016) and Barrena et al. (2018) studied PSZ1 sources based on the observational program ITP13-08. Later, a second long-time program was granted 128-MULTIPLE-16/15B (hereafter LP15) to study the PSZ2 sources. Streblyanska et al. (2019; hereafter Paper I) and this paper are the result of this last program. The main motivation of these follow-up campaigns was to identify and confirm optical cluster counterparts of unknown sources. We performed photometric and spectroscopic observations in order to study the optical richness and estimate velocity dispersion. We left the mass estimates for future works.

This paper is structured as follows. Sections 2 and 3 describe the observational program carried out for this work, including the instrumentation setups, the observational approach, and the criteria we used to validate candidates as optical counterparts for SZ sources. Section 4 presents the observations for the second year of this program and details some special cases. In Sect. 5 we update information about already known counterparts that were validated using ALLWISE (Cutri et al. 2013). Section 6

\footnotetext{
2 The NASA/IPAC Extragalactic Database (NED) is operated by the Jet Propulsion Laboratory, California Institute of Technology, under contract with the National Aeronautics and Space Administration.
}

summarizes the whole program and gives the final results for this work. Section 7 presents the conclusions.

We adopt an $\Lambda$ CDM cosmology with $\Omega_{\mathrm{m}}=0.3075, \Omega_{\Lambda}=$ 0.691 , and $H_{0}=67.74 \mathrm{~km} \mathrm{~s}^{-1} \mathrm{Mpc}^{-1}$.

\section{LP15 optical follow-up campaign}

\subsection{Sample definition and observational strategy}

The LP15 program, the sample definition, and the observing strategy are described in detail in Paper I. Here, we briefly summarize its basic characteristics and present the second year of observations. We recall that the main motivation of the program is to carry out a systematic follow-up of the complete set of PSZ2 cluster candidates in the northern sky that had no confirmed counterparts at the moment of the catalog publication. For LP15, we considered only sources located at a declination higher than $-15^{\circ}$. As a reference, the full PSZ2 catalog contains a total set of 1003 sources in this region.

The LP15 sample is defined by all sources in the PSZ2 with a declination higher than $-15^{\circ}$ that also have a validation $=-1$ (i.e., no known counterpart at the time of the publication of the catalog), and PSZ1 $=-1$ (i.e., no matching detection in the PSZ1). This corresponds to 190 targets in total, 106 of which have previously been discussed in Paper I.

During the second year of the program, covering the semesters 2016B and 2017A, we continued with the observations of the remaining LP15 sample. As for the previous year, the observations in the second year were carried out at the Roque de los Muchachos Observatory (ORM) located at La Palma island (Spain) between August 2016 and August 2017. The three telescopes used in this work are (a) the $2.5 \mathrm{~m}$ Isaac Newton Telescope (INT) operated by the Isaac Newton Group of Telescopes, (b) the $3.6 \mathrm{~m}$ Italian Telescopio Nazionale Galileo (TNG) operated by the Galileo Galilei Foundation of the Istituto Nazionale di Astrofisica (INAF), and (c) the $10.4 \mathrm{~m}$ Gran Telescopio Canarias (GTC) operated by the Instituto de Astrofísica de Canarias (IAC). More information about the instruments and technical features has been presented in Table 1 of Paper I, together with information about the observing nights and the number of candidates that were observed.

Our follow-up program was structured as follows. The first step was to select a target for deep-imaging observations. When galaxy overdensities were found, the cluster was confirmed using spectroscopy. The photometric observations were carried out in the INT using three broadband Sloan filters $\left(g^{\prime}\right.$, $\left.r^{\prime}, i^{\prime}\right)$. Based on color combinations of these filters, we were able to estimate photometric redshifts (Planck Collaboration Int. XXXVI 2016) of possible members of the cluster up to $z \sim 0.8$. In the second step, candidates were unambiguously confirmed using multi-object spectroscopy (MOS) either in the TNG or in GTC, depending on the photometric estimation of the redshift of the candidate. Because the collecting area of the GTC is greater than that of the other telescopes, we used this for distant clusters $(z>0.35)$, while the TNG was used for the closest clusters $(z<0.35)$. The final step was to validate the candidates using the confirmation criteria explained in Sect. 3.

\subsection{Imaging and spectroscopic observation and data reduction}

The complete technical description of the telescopes and instrument setups used during the LP15 program and information on corresponding data reduction is detailed in Paper I. Here, we 

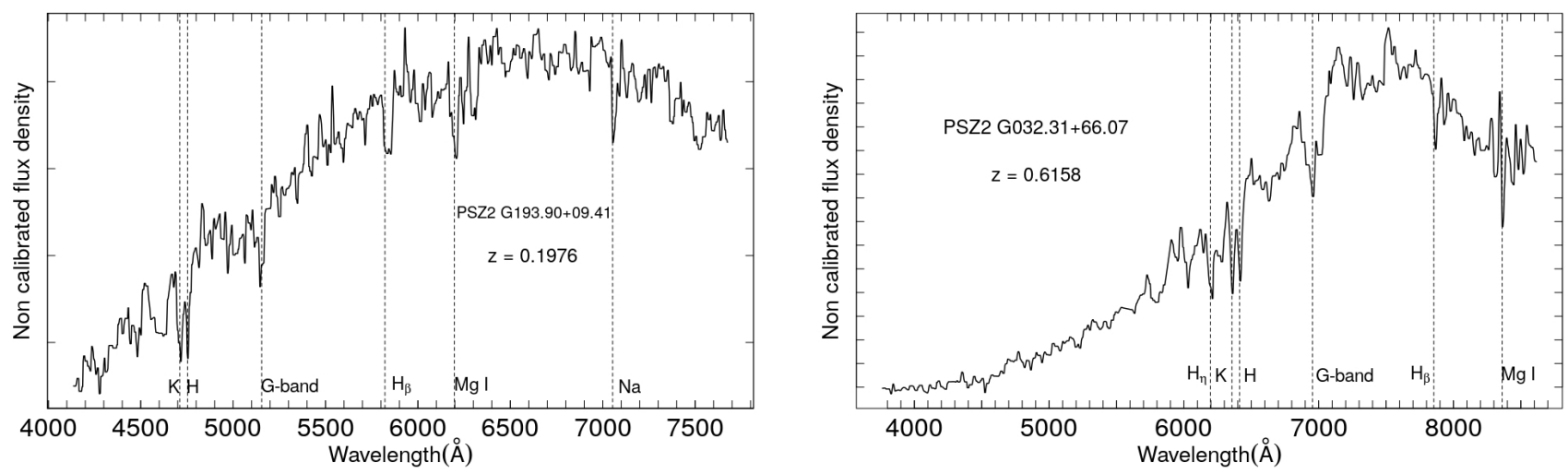

Fig. 1. Example of the spectra obtained with TNG/DOLORES (left panel) and GTC/OSIRIS (right panel) for two luminous galaxy members in the PSZ2 G193.90+09.41 and PSZ2 G032.31+66.07 clusters at $z=0.1976$ and $z=0.6158$, respectively. Dashed lines correspond to the wavelength of the absorption features that were identified in each spectrum at the redshift of the clusters. The flux density is plotted in arbitrary units.

briefly summarize the information of the imaging and spectroscopic observations and data reduction that were performed during the second year of the program.

Optical observations were carried out during multiple runs between August 2016 and August 2017. Deep images were obtained using the Wide-Field Camera (WFC) installed at the $2.5 \mathrm{~m}$ of the INT. Spectroscopic observations were obtained using the multi-object spectrographs Device Optimized for the LOw RESolution (DOLORES) at the TNG and Optical System for Imaging and low-Intermediate-Resolution Integrated Spectroscopy (OSIRIS) at the GTC. The spectroscopic data from DOLORES were retrieved during multiple runs between November 2016 and August 2017 and the data from OSIRIS were acquired in service mode between September 2016 and August 2017.

All images and spectra were reduced using $\operatorname{IRAF}^{3}$ standard routines. The astrometry was performed using the images.imcoords task and the USNO B1.0 catalog (Monet et al. 2003) as reference. From this we obtained a final accuracy of rms $\sim 0.2^{\prime \prime}$ across the full field of view. The photometric calibration is referred to the SDSS photometry. In the case of fields with SDSS coverage, we performed the direct cross correlation with the SDSS photometric data. Using the SExtractor (Bertin \& Arnouts 1996) program in single-image mode, we created individual source catalogs for each band. All catalogs were then merged into the final catalog with a search radius of $1^{\prime \prime}$.

Standard spectroscopic data reduction included sky subtraction, extraction of spectra, cosmic-ray rejection, and wavelength calibration (using specific arcs). Our final reduced spectra typically have $S / N \sim 5$ per pixel (at around $6000 \AA$ ) for galaxies with magnitudes $r^{\prime} \sim 20.5$ and 21.7 observed with the TNG and GTC, respectively.

We estimated radial velocities using the task $\mathrm{RVSAO}^{4}$. Figure 1 shows two examples of spectra for low- and high- $z$ galaxies obtained at the TNG and GTC. Based on the capabilities of the MOS observations, we were able to typically retrieve 20 members per cluster with which to estimate the mean redshift and velocity dispersion of the systems. The cluster redshift was taken to be the mean value of the galaxy members that we retrieved. The galaxies were considered members only when they showed

3 IRAF (http://iraf.noao.edu/) is distributed by the National Optical Astronomy Observatories, which are operated by the Association of Universities for Research in Astronomy, Inc., under the cooperative agreement with the National Science Foundation.

4 RVSAO was developed at the Smithsonian Astrophysical Observatory Telescope Data Center. radial velocities of $\pm 2500 \mathrm{~km} \mathrm{~s}^{-1}$ in rest frame with respect to the mean velocity of the system. Then, we followed an iterative method considering galaxies as members when their radial velocity was lower than 2.5 times the velocity dispersion away from the cluster mean velocity. We followed this procedure in order to minimize contamination by interlopers. The velocity dispersion and mass of the clusters will be published in a future work.

The broadband images we used have previously been included in the Virtual Observatory (VO) collection for public access. In the near future, the photometric and spectroscopic catalogs will also be available through this platform.

\section{Cluster identification and validation criteria}

Here we describe the method we used to validate a cluster candidate as the optical counterpart of a PSZ2 target. This procedure is similar to the one adopted in Paper I, and it is an extension of the method applied in Planck Collaboration Int. XXXVI (2016), Barrena et al. (2018). Compared to other methods in the literature, we have improved the validation criteria by including visual inspection and comparison between the RGB images, and also the Compton $y$-maps (Planck Collaboration XXII 2016), making photometric redshift estimates and analyzing the red sequence (hereafter RS Gladders \& Yee 2000) using color-magnitude diagrams. We also performed a richness study considering galaxy counts in clusters. For approximately $30 \%$ of our sample we performed spectroscopic confirmation by estimating the velocity dispersion of the candidates.

\subsection{Photometric analysis}

The first step in our validation process was to visually inspect the deep RGB images and compare them with the Compton $y$ map of the SZ source. With the nominal Planck pointing coordinates as reference, we searched for galaxy overdensities inside a region of about $5^{\prime}$ radius, which is 2.5 times the Planck mean position error (Planck Collaboration XXVII 2016). However, when a possible counterpart was found farther away than $5^{\prime}$, we performed a more detailed analysis in a few cases. In this first step we stress that we identified galaxy overdensities in the field of view but not every overdensitiy was associated with the SZ source. The next steps were performed in order to study this possible association.

We used color-magnitude diagrams to identify likely cluster members and fit the RS to estimate the photometric redshift using the method explained in Barrena et al. (2018), Sect. 3 and 


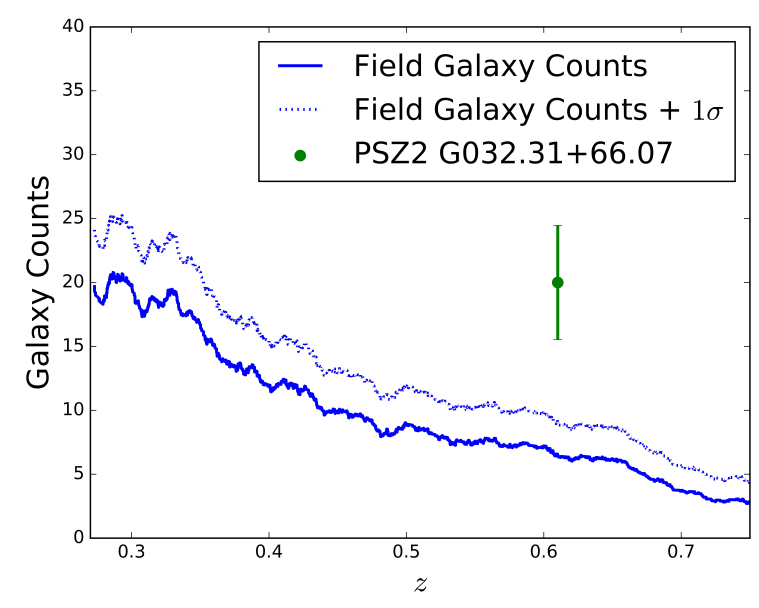

Fig. 2. Galaxy counts as a function of redshift in the field of the spectroscopically confirmed cluster PSZ2 G032.31+66.07. The galaxy counts for this particular cluster and its $1 \sigma$ error bars are shown in green. The blue line represents the galaxy counts outside the $1 \mathrm{Mpc}$ region from the optical center of the cluster, and the dashed blue line represents $1 \sigma$ uncertainty above the latter.

in Planck Collaboration Int. XXXVI (2016), Sect. 4.2. To do so, we used $\left(g^{\prime}-r^{\prime}\right)$ and $\left(r^{\prime}-i^{\prime}\right)$ colors and considered galaxies within \pm 0.05 from the color of the brightest cluster galaxy (BCG).

The SZ clusters of the Planck catalog are expected to be massive structures $\left(\gtrsim 5 \times 10^{14} M_{\odot}\right.$ ) (see Planck Collaboration XXIX 2014) and are therefore thought to present rich galaxy cluster populations. In order to discard the low-mass systems from the real massive counterparts, we defined a richness parameter $\left(R_{0}\right)$ and its significance above the background level $\sigma_{\mathrm{R}}$. A detailed description of our richness calculations is given in Paper I. Briefly, for a given cluster, we counted likely cluster members (assumed to be galaxies within the $\mathrm{RS} \pm 0.15$, in color) with $r^{\prime}$ magnitudes in the range $\left[m_{r^{\prime}}^{\star}-1, m_{r^{\prime}}^{\star}+1.5\right]$ within the $1 \mathrm{Mpc}$ from the cluster center. For this task, we considered as a BCG the most luminous galaxy of the identified likely cluster members. In order to obtain the background-subtracted richness and remove the galaxy field contribution, we computed galaxy counts outside the $1 \mathrm{Mpc}$ region using the same restrictions in color and magnitude as explained before, thereby creating a field galaxy sample or local background for each cluster $\left(R_{\mathrm{f}}\right)$. This field sample was scaled to the $1 \mathrm{Mpc}$ area and subtracted from the cluster counts to obtain a statistical estimate of the number of galaxies for each cluster, the socalled richness $\left(R_{\text {cor }} \equiv R_{0}-R_{f}\right)$. We based our confirmation on the significance above the background level $\sigma_{\mathrm{R}}$, which is computed as $R_{\text {cor }} / \sqrt{R_{\mathrm{f}}}$. For a better understanding, see Fig. 2, which shows galaxy counts as a function of redshift in the field of the confirmed cluster PSZ2 G032.31+66.07. The initial value found for this cluster was $R_{0}=20.0$, and for the field at the redshift of the cluster, it was $R_{\mathrm{f}}=6.4$, yielding $R_{\text {cor }}=13.6$ and $\sigma_{\mathrm{R}}=5.4$.

\subsection{Spectroscopic analysis}

We performed spectroscopic observations for approximately $30 \%$ of our sample, and include public data from the SDSS spectroscopic archive. We built radial velocity catalogs for each cluster and obtained the velocity dispersion in order to distinguish between poor and massive systems.

Planck systems must have masses $M_{500} \gtrsim 10^{14} M_{\odot}$ at low redshift $(z<0.2)$ and $M_{500} \gtrsim 2 \times 10^{14} M_{\odot}$ at higher redshift (see van der Burg et al. 2016, Fig. 4). Using the scaling relations $M_{500}-\sigma_{\mathrm{v}}$ from Munari et al. (2013) and $M_{500}-M_{200}$
Table 1. Validation criteria adopted to confirm or reject candidates associated to the SZ emission.

\begin{tabular}{lccc}
\hline \hline Flag & Spectroscopy & $\sigma_{\mathrm{v}}\left(\mathrm{km} \mathrm{s}^{-1}\right)$ & $\sigma_{\mathrm{R}}$ \\
\hline 1 & Yes & $>500 \mathrm{~km} \mathrm{~s}^{-1}(z<0.2)$ & $>1.5$ \\
& & $>650 \mathrm{~km} \mathrm{~s}^{-1}(z>0.2)$ & $>1.5$ \\
\hline 2 & No & - & $>1.5$ \\
\hline 3 & Yes & $<500 \mathrm{~km} \mathrm{~s}^{-1}(z<0.2)$ & $>1.5$ \\
& & $<650 \mathrm{~km} \mathrm{~s}^{-1}(z>0.2)$ & $>1.5$ \\
& No & - & $<1.5$ \\
\hline ND & - & - & - \\
\hline
\end{tabular}

from Komatsu et al. (2011), these mass values lead to a velocity dispersion of $\sigma_{\mathrm{v}}>500 \mathrm{~km} \mathrm{~s}^{-1}$ for $z<0.2$ and $\sigma_{\mathrm{v}}>650 \mathrm{~km} \mathrm{~s}^{-1}$ for $z \geq 0.2$. We adopt here the same criteria as were used in previous validation studies of Planck clusters (Planck Collaboration Int. XXXVI 2016; Barrena et al. 2018; Streblyanska et al. 2019). The velocity dispersion of the candidates will be presented in a future work.

\subsection{Confirmation criteria adopted in this work}

Table 1 summarizes the set of criteria we adopted in order to confirm or reject a cluster candidate as the optical counterpart of the SZ signal. These are the very same criteria as were used in Paper I, and they provide a classification of the candidates according to four possible values of a Flag. Values of Flag = 1 or 2 correspond to validated clusters, Flag $=3$ corresponds to clusters located along the line of sight of the Planck signal but that may not be associated with the SZ emission, and ND refers to a nondetection. When we had enough spectroscopic information to provide an estimate of $\sigma_{\mathrm{v}}$, we validated the candidate with Flag 1 if that value was found to be above the corresponding threshold. However, when $\sigma_{\mathrm{v}}$ was below the threshold, we assumed that the system has a low mass and is probably not linked to the SZ emission. These candidates are classified as Flag 3. When no spectroscopic information is available, or when we were unable to estimate the velocity dispersion due to an insufficient number of galaxy members (fewer than five members), we validated the candidates using the richness estimate. Systems with $\sigma_{\mathrm{R}}>1.5$ were validated photometrically, but still await a definitive spectroscopic confirmation. These systems are classified with Flag 2. Clusters with Flag 3 represent very poor systems $\left(\sigma_{\mathrm{R}}<1.5\right)$ without spectroscopic information. The ND flag was used for SZ candidates without a galaxy overdensity in the optical images. We also considered the criterion that a Planck cluster must be placed within a $5^{\prime}$ radius from the nominal pointing because it represents 2.5 times the mean position error with respect to the SZ peak emission. Nevertheless, this criterion can be modulated due to the wide range of uncertainties in the position error in the PSZ2 catalog and the different shapes of the $y$-maps. The cases that do not match the validation criteria but are positively confirmed are discussed in Sect. 4.2.

\section{LP15 sample: second year of observations}

\subsection{Results of the second year of observations}

Table 2 summarizes the basic information of program LP 15 after the two years of observations for the characterization of the LP15 sample. The results for year one of the program have previously been discussed in Paper I. Here, Table A.1 presents the results 
Table 2. Summary information of the long-term LP15 program.

\begin{tabular}{lccccccc}
\hline \hline Year & Observed & Val & Spec & Flag 1 & Flag 2 & Flag 3 & ND \\
\hline 1 & 106 & 41 & 34 & 31 & 10 & 8 & 57 \\
2 & 78 & 40 & 22 & 18 & 22 & 6 & 32 \\
\hline Total & 184 & 81 & 56 & 49 & 32 & 14 & 89 \\
\hline
\end{tabular}

Notes. For each year of the program, we show the total number of observed candidates (Col. 2), the total number of validated clusters (Col. 3), and the fraction of those with spectroscopic measurements (Col. 4). For completeness, Cols. 5-8 also include the classification of the candidates according to our validation criteria described in Table 1. Validated clusters (Col. 3) are those with Flags 1 or 2 . The full LP15 sample contained 190 candidates. This means that 6 objects remain to be studied (see text for details).

for 78 PSZ2 galaxy cluster candidates studied in this optical follow-up during the second and last year. The table is organized as follows. Columns 1,2 , and 3 are the official ID number, the Planck name and the SZ signal-to-noise ratio, respectively, as they appear in the PSZ2 catalog. Columns 4 and 5 are the J2000 coordinates of the BCG when present, otherwise, the geometrical center of the cluster is provided. Column 6 is the distance between Planck and the optical center reported in this work. Columns 7 and 8 present the spectroscopic information when available: the mean spectroscopic redshift of the cluster and/or the BCG, and the number of spectroscopic members retrieved. Columns 9, 10, and 11 provide the photometric information: the photometric redshift, the richness estimate and the value $\sigma_{\mathrm{R}}$ as explained in Sect. 3.1. Column 12 lists the cluster classification following the Flag system described in Sect. 3.3. Finally, Col. 13 is reserved for special comments or notes about the individual candidates.

Following the confirmation criteria explained in Sect. 3.3, we find that 37 of our candidates have a single optical counterpart, and one additional source is classified as a double detection. We classified a source as a double detection when two or more overdensities were found around the SZ emission peak that might contribute to this emission. Those that were validated with a single optical counterpart are classified as follows: 17 have Flag 1 and 22 have Flag 2. In addition, we find 32 nondetections, flagged ND, and 6 systems that are not associated with the corresponding SZ source (Flag 3). This means that a total of 38 PSZ2 sources remain unconfirmed.

We have partially focused our work using SDSS DR12 data to confirm PSZ2 clusters that were classified by Streblyanska et al. (2018) as "potentially associated" with the SZ emission. We obtained the redshift and the velocity dispersion for six of the photometrically confirmed clusters and we reconfirmed six clusters using our own deep INT imaging data by classifying them as Flag 1 and 2, respectively. From the "potentially associated" sub-sample of Streblyanska et al. (2018) we confirmed five as Flag 1. Here, we invalidate the PSZ2 G328.96+71.97, confirmed by Streblyanska et al. (2018). New SDSS DR14 data reveal that the counterpart proposed by the authors is part of a larger system whose BCG is 34.'6 away from the Planck SZ pointing. This system is discussed in detail in Sect. 4.2.

Figure 3 represents the spatial distribution of the optical counterpart center with respect to the nominal center in the Planck PSZ2 catalog for the clusters in our sample flagged 1, 2 , and 3 . The median of the offsets of the clusters validated is 3 .'10 which is in agreement with the median of the position error computed from the PSZ2 catalog (2!43). The mean values are also in agreement (3.3 and 2.6), but they are more sensitive to cases whose position error is too high. We find 25 (35) clusters within $4.46(6.38)$, representing $68 \%(95 \%)$ of the confirmed clusters.

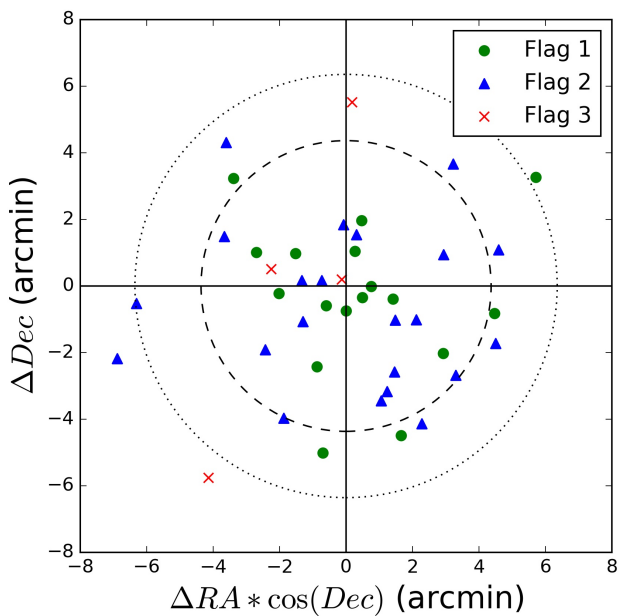

Fig. 3. Spatial distribution of the optical counterpart centers with respect to the nominal center in the Planck PSZ2 catalog. Green dots, blue triangles, and red crosses correspond to clusters classified with Flag 1, Flag 2, and Flag 3, respectively. Dashed and dotted lines show the regions enclosing $68 \%$ and $95 \%$ of the confirmed clusters, flagged 1 and 2 , respectively

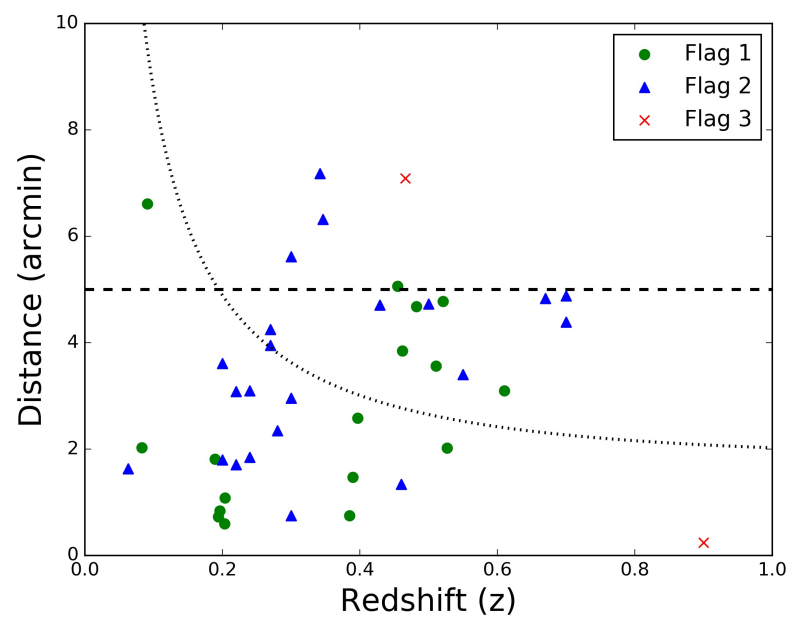

Fig. 4. Cluster optical center offsets relative to their Planck SZ position as a function of cluster redshift. The dashed horizontal line at $5^{\prime}$ shows the maximum offset expected for a Planck SZ detection. The dotted line corresponds to the angle subtended by $1 \mathrm{Mpc}$ in projection at the corresponding redshift. The symbols are the same as in Fig. 3.

Figure 4 shows the cluster optical center offsets relative to their Planck SZ position as a function of cluster redshift. Although the limit in distance is $5^{\prime}$ (see Sect. 3), some confirmed sources exceed this value for different reasons such as the high 


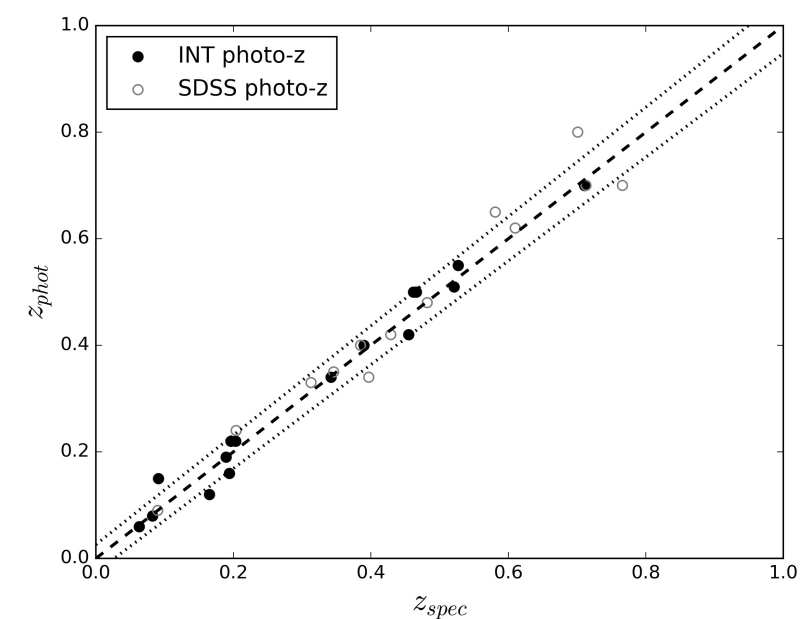

Fig. 5. Comparison between photometric and spectroscopic redshift estimates. The dashed line represents the 1:1 relation, and the dotted lines show the photometric redshift error $\delta z /(1+z)=0.026$.

position error in the PSZ2 catalog or elongated $y$-map contour around the SZ emission peak (see Sect. 4.2).

Figure 5 compares photometric and spectroscopic redshift estimates. Photometric redshifts were obtained as explained in Sect. 2. We show every observation carried out during the second year of the LP15, including those explained in Sect. 5. This study yields a mean photometric redshift error of $\delta z /(1+z)=0.026$.

Finally, we note that six objects in the LP15 sample have not been observed during the program. One of them, PSZ2 G186.50-13.45, has been validated in Streblyanska et al. (2018), with a photometric redshift of $z_{\text {phot }}=0.25$. According to our validation criterion, this case would correspond to a $\mathrm{Flag}=2$.

A dedicated proposal has been submitted and approved to complete the observations for the remaining five objects PSZ2 G023.05+20.52, PSZ2 G092.34+14.22, PSZ2 G206.55-43.22, PSZ2 G210.37-37.00, and PSZ2 G247.14+25.88. These observations were going to be conducted in July 2019. Due to bad meteorology and technical problems we were not able to perform the observations. We plan to submit a new proposal in the near future.

\subsection{Notes on individual objects}

PSZ2 G058.31+41.96 This candidate is flagged as a nondetection because a bright star is located near the Planck pointing. The star prevents photometric measurements of this region, and we are therefore unable to visually identify an overdensity of galaxies. Despite this problem, we cannot identify visually any overdensity of galaxies in the region.

PSZ2 G104.52+39.39. The distance from the optical center and the Planck nominal pointing is 5.62. Nevertheless, we validate this cluster with Flag 2 because the MILCA $y$-map contours are elongated along the line that links the optical and Planck centers. In addition, the position error in the Planck catalog is too high (5'40) compared to the nominal error (2.'43).

PSZ2 G130.64+37.16. This candidate has two optical counterparts that have been validated in Streblyanska et al. (2018); one was also validated by Burenin (2017). This is a difficult case as we show in Fig. 6. For a detailed description see caption of Fig. 6.

PSZ2 G146.13+40.97. The optical center of the proposed counterpart (its BCG) is 7.'18 away from the Planck SZ pointing,

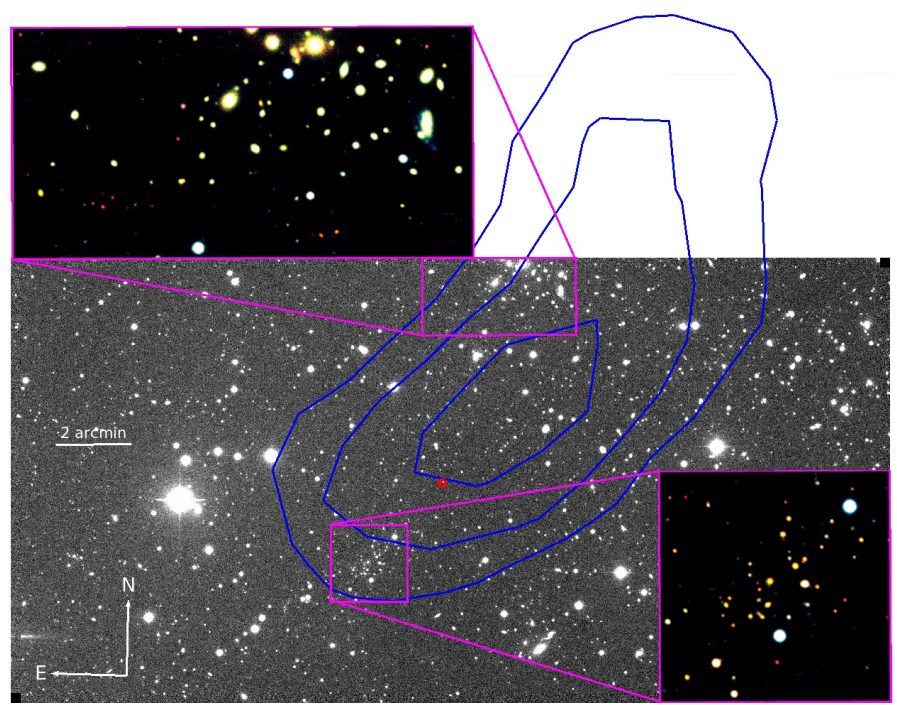

Fig. 6. Compton $y$-map superimposed on the INT $r^{\prime}$ band of PSZ2 G130.64+37.16. Blue contours correspond to the 4, 4.6, and $5.2 \times 10^{-6}$ levels of the Compton $y$-map in this area. The nominal SZ pointing (red) is clearly closer to cluster 644-A (zoomed-in in the lower right region), which has 14 spectroscopic members and shows a velocity dispersion close to $1000 \mathrm{~km} \mathrm{~s}^{-1}$; cluster 644-B (zoomed-in in the upper left region) is 6.07 away from the SZ center, but the MILCA $y$-map shows that the contours are elongated along this counterpart location, which is twice as rich as that of 644-A. The position error in the Planck catalog here is 5.38 , which is more than twice the mean position error.

which is affected by a position error of 5.89. The $y$-map contours present a very irregular shape, possibly as a result of galactic dust contamination in this region. We estimate a richness of $\sigma_{\mathrm{R}}=3.5$ for this system and find four cluster members at $z_{\text {spec }}=0.342$ in the SDSS DR14 spectroscopic sample. We therefore classify this counterpart with $\mathrm{Flag}=2$. The ultimate confirmation will be obtained using MOS observations.

PSZ2 G152.47+42.11. We find a possible cluster counterpart at $z_{\text {spec }}=0.900$. The deepness of our images makes it impossible to estimate the richness at this redshift. However, we have observed this system spectroscopically and found six cluster members. From these six galaxies, we obtain a very lowvelocity dispersion $\left(<400 \mathrm{~km} \mathrm{~s}^{-1}\right)$, revealing a low-mass galaxy system. In this case, we therefore classify this optical counterpart with Flag $=3$.

PSZ2 G156.24+22.32. This region encloses two very bright stars, which makes it very difficult to obtain accurate photometry or richness. However, by eye inspection, we identified a cluster with a galaxy population with coherent colors. A detailed study of the photometry of some individual likely members and the BCG revealed $z_{\text {phot }}=0.30$. In addition, the $y$-map contours present a very regular profile centered on this system. For all these reasons, we classify this system with Flag $=2$.

PSZ2 G177.03+32.64. Burenin (2017) reported a counterpart for this candidate at $z \sim 0.28$. We analyzed this overdensity and found seven galaxies with spectroscopic redshifts in the SDSS archive. Four of these galaxies are more than $4 \mathrm{Mpc}$ away from the Planck center, and the velocity dispersion accounting for the seven galaxies is lower than $300 \mathrm{~km} \mathrm{~s}^{-1}$. For this reason, we present here only one counterpart at $z_{\text {spec }}=0.511$ whose velocity dispersion, calculated using nine members, is approximately $1000 \mathrm{~km} \mathrm{~s}^{-1}$.

PSZ2 G183.92+16.36. The distance between the BCG of this cluster and the Planck pointing is 6.'61, which is only $0.67 \mathrm{Mpc}$ 
at the redshift of the cluster, $z_{\text {spec }}=0.091$ (see Fig. 4). We performed multi-object spectroscopy and retrieved 18 cluster members, showing $\sigma_{\mathrm{v}} \sim 650 \mathrm{~km} \mathrm{~s}^{-1}$. In addition, this cluster is known as Abell 567. It is well consolidated by other observations in the past (Abell et al. 1989). We therefore confirm Abell 567 as the counterpart of this SZ source and classify it with $\mathrm{Flag}=1$.

PSZ2 G202.61-26.26 and PSZ2 G203.32+08.91. Both candidates were analyzed using PANSTARRS photometric data (Chambers et al. 2016). Both systems are rich, but the optical center of the first lies more than $5^{\prime}$ away from the Planck nominal pointing, therefore it was classified with Flag 3.

PSZ2 G227.30+09.00. This is an SZ source placed at very low galactic latitude, so that many stars crowd this field. For this reason, we were unable to compute the richness: the galaxies of the background are partially masked by the foreground stars. However, this system presents X-ray emission and has been cataloged as 1RXS J075020.3-082605 in the ROSAT survey. We therefore classify this source with $\mathrm{Flag}=2$.

PSZ2 G237.68+57.83. This cluster has previously been validated by Streblyanska et al. (2018) using SDSS data. Here, we confirm this association using the INT images. Although we are unable to perform a richness estimation at this redshift, the photometry of some individual likely cluster members agrees with a $z_{\text {phot }}=0.70 \pm 0.05$. We also find two additional overdensities at $(\mathrm{RA}=10: 53: 35.55, \mathrm{Dec}=+10: 43: 45.71)$ and $(\mathrm{RA}=10: 53: 59.602, \mathrm{Dec}=+10: 46: 38.23)$. However, they are at a distance greater than $10^{\prime}$ from the SZ coordinates and therefore probably do not contribute to the SZ signal. Therefore we validated PSZ2 G237.68+57.83 as a single counterpart at $z_{\text {phot }}=0.70$.

PSZ2 G271.53+36.41. This candidate was confirmed photometrically in Streblyanska et al. (2018) as a double detection. However, only one cluster is visible in the INT images. This cluster is at $z_{\text {phot }}=0.50 \pm 0.03$. No more systems are associated with this SZ source.

PSZ2 G328.96+71.97 was validated by Streblyanska et al. (2018) using SDSS DR12 data. Here, we used new spectroscopic information provided by SDSS DR14 in order to update the information. We find 94 cluster members at $\left\langle z_{\text {spec }}\right\rangle=$ 0.090. However, the BCG of this structure is at 34.6 from the Planck pointing. Figure 7 shows the scenario around this region. The profile of the SZ emission is very spread out and irregular, with several peaks. The 94 cluster members have $\sigma_{\mathrm{v}} \sim 800 \mathrm{~km} \mathrm{~s}^{-1}$ and a virial radius of $1.6 \mathrm{Mpc}$, but the cluster seems to be placed completely off the SZ peak. The distance between the Planck pointing and the optical cluster center is larger (twice) than the virial radius of the cluster. For all these reasons, we conclude that no optical counterpart is found for this SZ source, and the actual counterpart (if it exists) is still unknown.

\section{Observations of other PSZ2 candidates beyond the LP15 sample}

In the PSZ2 catalog, 73 clusters are validated using the AllWISE mid-infrared source catalog, see Planck Collaboration XXVII (2016). This catalog is based on the observations from the Widefield Infrared Survey Explorer mission (WISE; Wright et al. 2010). In Sect. 7.4 in Planck Collaboration XXVII (2016), the authors searched for galaxy overdensities in the redshift range $0.3<z<1.5$ using the $(W 1-W 2)$ color. These objects had validation $=16$ in the original PSZ2 catalog, and were therefore not included in the definition of the LP15 sample.

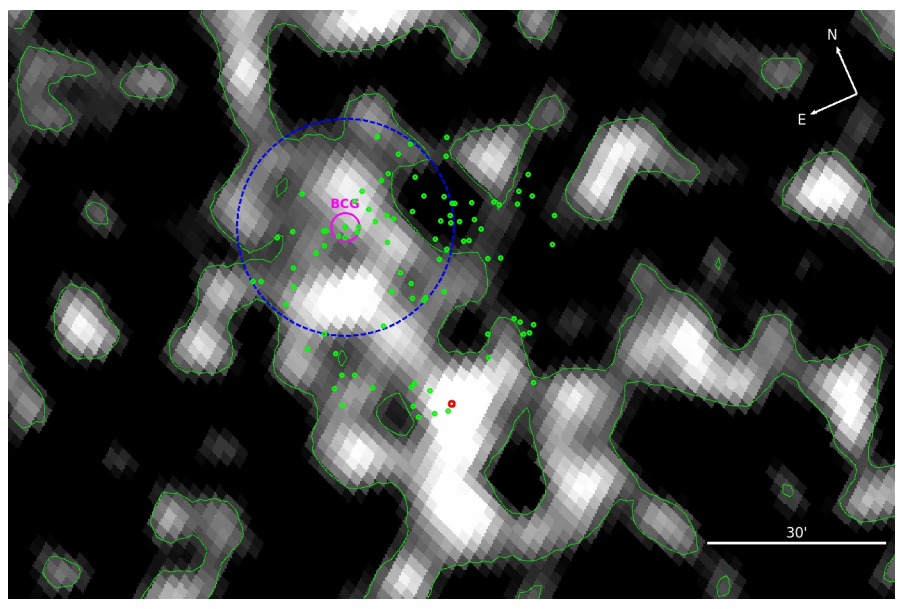

Fig. 7. SZ emission as seen in the Compton $y$-maps for the source PSZ2 G328.96+71.97. The red dot represents the SZ coordinate as it appears in the PSZ2 catalog. The BCG of the cluster is plotted in magenta, and the remaining galaxies members are shown in green. The blue circle encloses the virial radius of this cluster. The 94 cluster members are placed completely off the SZ peak, and the optical counterpart of this SZ source remains unknown.

\subsection{Results}

Here, we present an update on 38 of these ALLWISE sources and provide their spectroscopic redshifts, which were obtained using dedicated observations carried out with the telescope time within the LP15 program. Table A. 2 presents this information, and it is organized in the same way as Table A.1.

The double detections PSZ2 G086.28+74.76, PSZ2 G139.00+50.92, and PSZ2 G141.98+69.31 can be considered single detections even though secondary clusters are detected because they are very low-mass systems and are therefore not capable of contributing significantly to the SZ signal.

As noted in earlier by the Planck Collaboration, the number of double cluster detections is somewhat higher than in other surveys in X-rays (Planck Collaboration Int. I 2012) or in the optical (Planck Collaboration Int. XXVI 2015).

We cross-checked our sample with two galaxy cluster catalogs, Wen, Han and Liu (WHL; Wen et al. 2012) and Wen, Han and Yang (WHY; Wen \& Han 2018), which are based on optical and infrared data, respectively. The WHL catalog was published using SDSS data, while WHY used 2MASS (Skrutskie et al. 2006), WISE (Wright et al. 2010), and SuperCOSMOS (Hambly et al. 2001) data. We only find three matches with WHL. PSZ2 G076.55+60.29 and PSZ2 G141.98+ 69.31 are discussed in the next subsection together with PSZ2 G021.02-29.0, which is also part of the WHY catalog. We find seven matches with the WHY catalog. We agree at $1 \sigma$ in redshift except for PSZ2 G056.38+23.36. In this case, we estimate a photometric redshift of $z_{\text {phot }}=0.21 \pm 0.02$, while Wen $\&$ Han (2018) reported $z_{\text {phot }}=0.31 \pm 0.04$. This is compatible within $2 \sigma$.

We also present in this section an update on four sources that have been confirmed in the PSZ2 original catalog, Planck Collaboration XXVII (2016), because they were matched with PSZ1 clusters but without a redshift estimate. Here, we provide the photometric redshift for three of them and invalidate the previously confirmed PSZ2 G198.73+13.34, for which we were unable to find any galaxy overdensity. In a future publication, we will discuss this type of source, which we believe is a false validation. These four sources can be found in Table 3 . 
Table 3. Update of already known optical counterparts from the PSZ1.

\begin{tabular}{lcccccccccc}
\hline \hline ID & Planck name & SZ S/N & RA & Dec & Dist. (') & $z_{\text {phot }}$ & $R_{\text {cor }}$ & $\sigma_{\mathrm{R}}$ & Flag & PSZ1 name \\
\hline 897 & PSZ2 G196.65-45.51 & 4.91 & 034254.40 & -084107.70 & 1.52 & $0.25 \pm 0.03$ & - & - & 2 & PSZ1 G196.62-45.50 \\
901 & PSZ2 G198.73+13.34 & 6.03 & - & - & - & - & - & - & ND & PSZ1 G198.67+13.34 \\
1130 & PSZ2 G249.14+28.98 & 5.96 & 094457.60 & -134811.22 & 1.16 & $0.15 \pm 0.03$ & $18.6 \pm 4.3$ & 5.3 & 2 & PSZ1 G249.14+28.98 \\
1539 & PSZ2 G326.73+54.80 & 5.92 & 134514.70 & -053204.00 & 3.91 & $0.46 \pm 0.05$ & $20.3 \pm 4.5$ & 10.6 & 2 & PSZ1 G326.64+54.79 \\
\hline
\end{tabular}

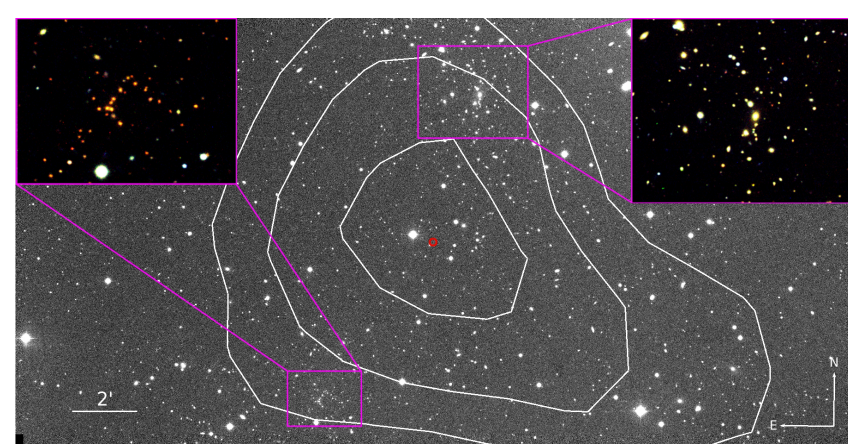

Fig. 8. Compton $y$-map superimposed to the INT $r^{\prime}$-band of the PSZ2 G076.55+60.29. White contours correspond to the 3, 4, and $5 \times 10^{-6}$ levels of the Compton $y$-map in this area. The Planck nominal pointing is represented in red. In the upper corners we show zoomed RGB images of 327-A (left) and 327-B (right). At $z_{\text {spec }}=0.632$ and $z_{\text {spec }}=0.287$, the two systems are associated with this SZ signal. This is a clear example of a multiple detection.

\subsection{Discussion on special cases}

We found that PSZ2 G076.55+60.29, which was classified as an individual counterpart by Streblyanska et al. (2018), is in fact a superposition of two clusters at $z_{\text {spec }}=0.287$ and $z_{\text {spec }}=0.632$, respectively. The first (327-A) has been proposed as a potentially associated cluster. Here, we confirmed it with five spectroscopic members. The distance to the Planck nominal pointing of the second counterpart (327-B) is slightly greater than $5^{\prime}$, but the MILCA $y$-map contours superimposed on an INT image (Fig. 8) show that the SZ emission is clearly a superposition of both clusters. The two counterparts are two of the richest systems we studied and have $\sigma_{\mathrm{R}}$ of 23.1 (327-A) and 8.4 (327-B).

PSZ2 G086.28+74.76. We find two clusters around the SZ emission at $z_{\text {spec }}=0.246$ and $z_{\text {spec }}=0.701$ that we call 381-A and 381-B, respectively. They have a high-velocity dispersion. However, the center of 381-A is 8.93 away from the Planck center. We therefore conclude that this source has only one optical counterpart at $z_{\mathrm{spec}}=0.701$.

PSZ2 G126.57+51.61 is one of the most distant clusters in our sample, at $z_{\mathrm{spec}}=0.816$. Burenin et al. (2018) confirmed one galaxy at $z_{\text {spec }}=0.815$. This cluster is at the detection limits of our deep optical images. Most of the members are detected almost at the noise level of the $i^{\prime}$-band image. No RS for this cluster could be constructed. However, inspection of the RGB image revealed an overdensity of red sources close to the Planck position supported by the contours extracted from the MILCA $y$-map (Fig. 9). Our spectroscopic data together with SDSS data confirm this cluster with 20 members and a $\sigma_{\mathrm{v}} \sim 850 \mathrm{~km} \mathrm{~s}^{-1}$.

PSZ2 G133.92-42.73. There is a potential counterpart, but it is discarded because of its low $\sigma_{\mathrm{R}}$. In the RGB image (left panel, Fig. 10) it seems to be a high-redshift cluster, as seen in the WISE image (right panel, Fig. 10). The SDSS data include three galaxies with $z_{\text {spec }} \sim 0.581$, but they are not associated

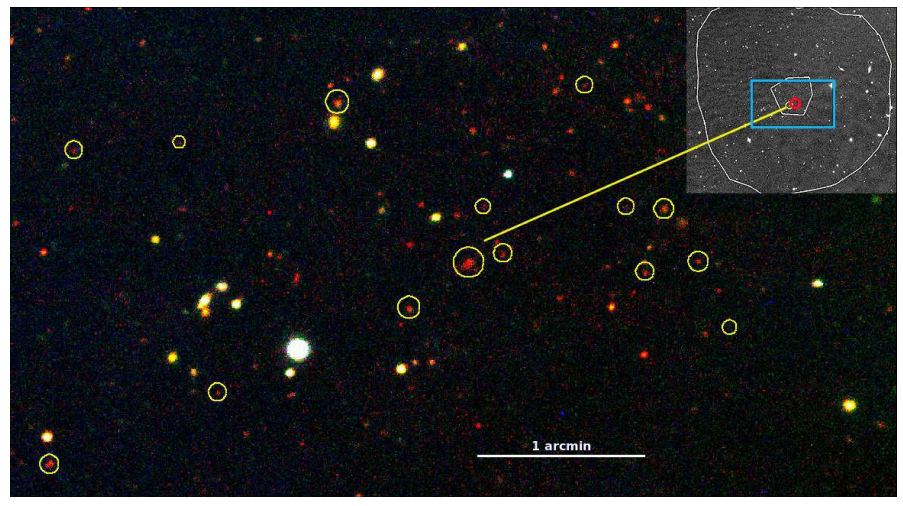

Fig. 9. Zoomed RGB image of the central area of the high- $z$ cluster $\left(z_{\text {spec }}=0.816\right)$ associated with PSZ2 G126.57+51.61. We mark spectroscopically confirmed cluster members with yellow circles. The small top panel shows the WFC/INT $i^{\prime}$-band image, in which white contours correspond to the 3 and $6 \times 10^{-6}$ levels of the Compton $y$-map in this area. The red circle indicates the nominal PSZ2 position. The blue square shows the size of the area that is presented in the main RGB image.

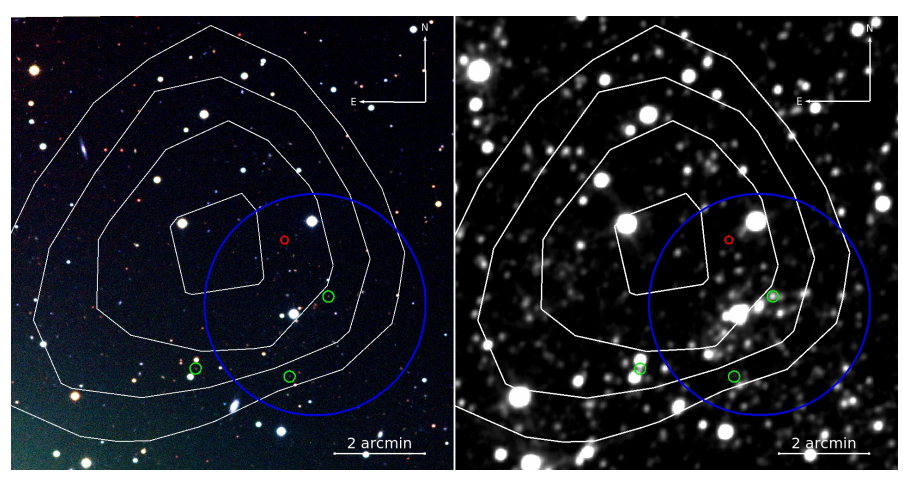

Fig. 10. Left: RGB image of the source PSZ2 G133.92-42.73. Right: WISE $W 1$ band of the same region. In both images Compton $y$-maps are represented in white $\left(5,5.8,6.6\right.$, and $7.4 \times 10^{-6}$ levels $)$. The blue region corresponds to $1 \mathrm{Mpc}(2.45)$ at the mean redshift of the three galaxies that are represented in green. The Planck nominal pointing is marked in red.

with any galaxy overdensity. Deeper imaging or spectroscopic observations are required in order to reject the possibility of a high $-z(z>0.8)$ cluster.

PSZ2 G139.00+50.92 has been confirmed by Streblyanska et al. (2018) at $z_{\text {phot }}=0.6$. We performed spectroscopic observations for this cluster, which we call 681-A, and found a velocity dispersion below the confirmation limit $\left(\sigma_{\mathrm{v}}<650 \mathrm{~km} \mathrm{~s}^{-1}\right)$. We conclude that this is not the main counterpart to the SZ emission. However, another cluster (681-B) at $z_{\text {spec }}=0.784$ has $\sigma_{\mathrm{v}}>800 \mathrm{~km} \mathrm{~s}^{-1}$, therefore we conclude that this last counterpart is responsible for the $\mathrm{SZ}$ emission. 


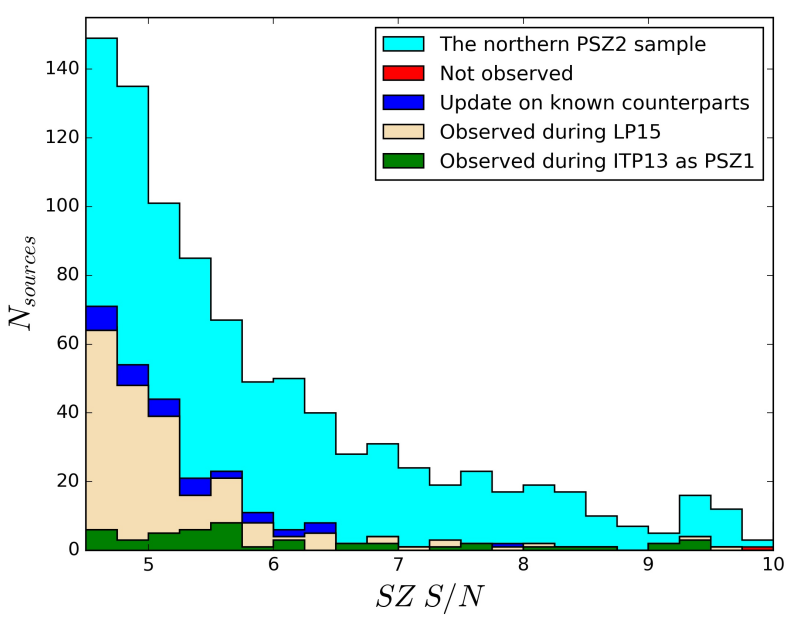

Fig. 11. PSZ2 cluster counts as a function of the $\mathrm{S} / \mathrm{N}$ of the $\mathrm{SZ}$ detection. The PSZ2-North sample is represented in light blue, the sources that are still not observed are represented in red $(<0.5 \%)$, the updated sources described in Sect. 5 are shown in dark blue (3.4\%), and the sources that were originally not confirmed and were observed during LP15 and ITP13 are shown in green ( $18.4 \%$ and $4.8 \%$, respectively). The bin size is 0.25 .

PSZ2 G141.98+69.31. This is a double detection. We find two overdensities in this field, but when we made spectroscopic observations and calculated the velocity dispersion, we realized that object $690-\mathrm{B}$ had $\sigma_{\mathrm{v}}<400 \mathrm{~km} \mathrm{~s}^{-1}$, which is very low for it to be associated with the SZ emission. Consequently, we only validate the object proposed in Streblyanska et al. (2018) with a spectroscopic redshift of $z_{\text {spec }}=0.713$, here called 690-A.

PSZ2 G270.78+36.83. This candidate has been validated by Streblyanska et al. (2018) as a double detection. Here, we spectroscopically confirm one of these counterparts by detecting 25 cluster members at $z_{\text {spec }}=0.516$, with $\sigma_{\mathrm{v}} \sim 900 \mathrm{~km} \mathrm{~s}^{-1}$. The second counterpart remains spectroscopically unconfirmed.

\section{PSZ2 statistics in the northern sky}

The PSZ2 catalog lists 1003 sources with Dec $>-15^{\circ}$. After the two years of LP15 observations, a total of 226 sources have been observed; 184 of them were part of the LP15 sample and thus were not validated at the time the PSZ2 catalog was published. In addition, we updated the redshift for 42 other sources. In this section, we carry out the statistical analysis of this northern sky subsample of the PSZ2 for purity and effects on the PSZ detection. For clarity, we refer to this subsample as PSZ2-North, which represents the $60 \%$ of the complete PSZ2 sample.

We note that this PSZ2-North sample also includes some PSZ2 sources that are associated with PSZ1 objects that were observed during the ITP13 (Planck Collaboration Int. XXXVI 2016; Barrena et al. 2018). Five sources $(<0.5 \%)$ could not be observed in order to validate the full PSZ2-North, therefore we exclude them form the sample when we compute the statistics in this section.

Figure 11 shows the number of clusters as a function of the $\mathrm{S} / \mathrm{N}$ in the catalog. The vast majority of the sources studied in this work has an $S / N<6$, and this is within the range where this optical follow-up found the largest contribution. In particular, we observed $37 \%$ of the sources with $4.5<S / N<6$.

We define the purity as the ratio between confirmed clusters and the total number of SZ sources. It is important to take into account that we have explored the optical range in which the dust

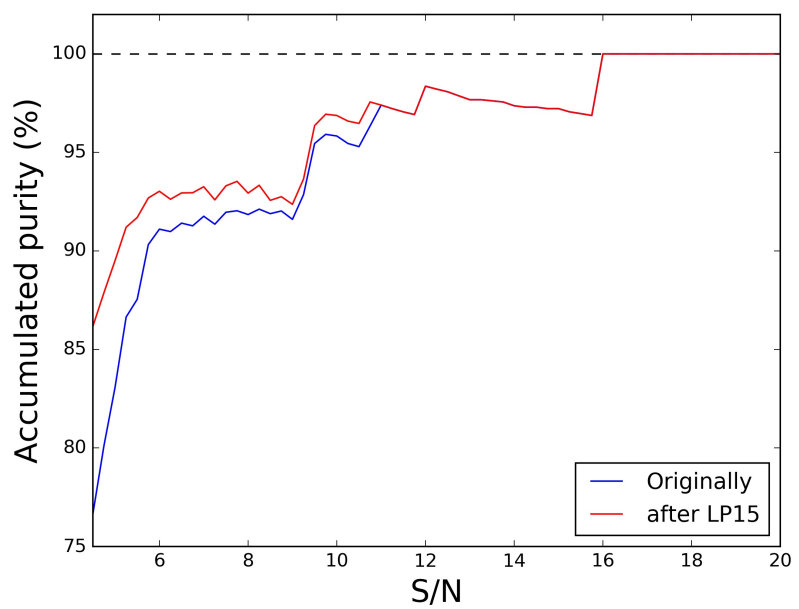

Fig. 12. Accumulated purity of the PSZ2-North sample (Dec $>-15^{\circ}$ ) studied as a function of the $\mathrm{S} / \mathrm{N}$, i.e., the percentage of the sources that are actual clusters and related to the SZ signal. In blue we represent the original purity of the catalog, and in red the same purity, but after this work.

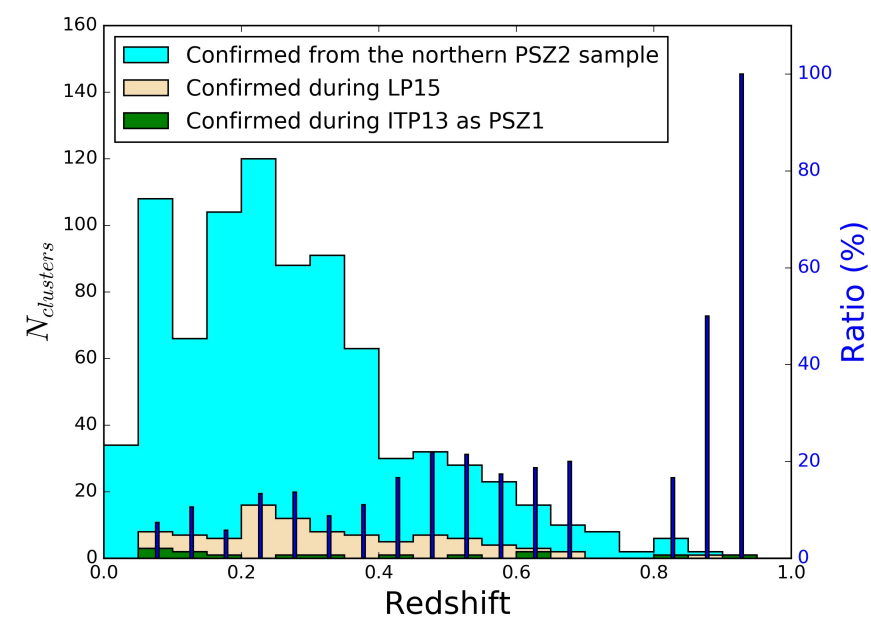

Fig. 13. Cluster counts as a function of redshift. Color codes are the same as in Fig. 11. Dark blue bars represent the ratio between clusters confirmed during our follow-ups and the total confirmed clusters. The size of the redshift bin is 0.05 .

emission might be masking the possible counterpart. We quantify this effect below. Figure 12 shows the accumulated purity of the PSZ2-North sample as a function of $S / N$. While it originally showed a purity of $76.7 \%$, the purity increased to $86.2 \%$ for $S / N>4.5$ after all validation programs. The feature in Fig. 12 that shows a decrease in purity in the range $12<S / N<16$ is due to one nondetection that is listed in the PSZ2 as a high $S / N$ source (PSZ2 G153.56+36.82). This has been studied in detail in Paper I.

Figure 13 shows the distribution of redshifts of the Planckconfirmed clusters. We note that $77 \%$ have a redshift between $0.05<z<0.4$, which is the ideal range for a cluster detection of the Planck mission. The median redshift of the PSZ2-North sample is 0.23 , and the median redshift of the clusters that were confirmed during LP15 is 0.29 . While we confirm about $10 \%$ of the clusters at $z<0.4$, this rate is $\sim 20 \%$ for $z>0.4$. Moreover, Barrena et al. (2018) confirmed the most distant Planck SZ cluster in the northern hemisphere: PSZ2 G123.35+25.39, at $z_{\text {phot }}=0.95$. 


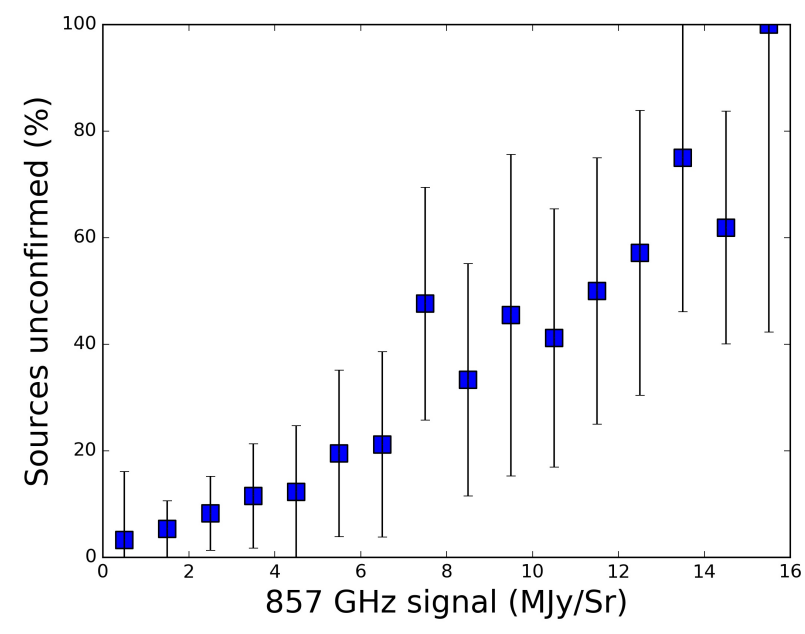

Fig. 14. Ratio between unconfirmed and total number of sources for the PSZ2-North sample (Dec $>-15^{\circ}$ ) as a function of the $857 \mathrm{GHz}$ signal in bins of $1 \mathrm{MJy} \mathrm{sr}^{-1}$. Error bars correspond to a Poisson error in the distribution of the total number of sources.

Burenin (2017) presented an extension for the PSZ2 cata$\log$ using SDSS and WISE. We find 28 matches in this cata$\log$ and the LP15 sample. Our results agree well for most of the sources. For PSZ2 G069.47-29.06 and PSZ2 G130.64+37.16, the author only reported one counterpart, while we find two. PSZ2 G069.47-29.06 was discussed in Paper I, where both candidates where confirmed with 44 and 30 spectroscopic members, as reported in Zaznobin et al. (2019). PSZ2 G130.64+37.16 was discussed in Sect. 4.2. In contrast, for PSZ2 G066.59-58.51, we find only one counterpart, while Burenin (2017) reported more than one.

We compared our results with those of Zohren et al. (2019). They used the WHT to validate high-z clusters of the Planck catalogs. They reported the redshift, richness, and mass for 23 candidates. Twenty of them were also observed during the LP15 program. We agree with their results except for three cases. They claimed, as did Burenin et al. (2018), that PSZ2 092.69+59.92 has two counterparts, at $z=0.46$ and $z=0.84$. Our spectroscopic observations reveal that the galaxy overdensity at $z=$ 0.84 is a low-mass system because it has $\sigma_{\mathrm{v}}<450 \mathrm{~km} \mathrm{~s}^{-1}$. They found that the mass limit of PSZ2 G139.00+50.92 lay below their validation limit. As discussed in Sect. 5.2, we find two possible counterparts, one (681-B) with $\sigma_{\mathrm{v}}>800 \mathrm{~km} \mathrm{~s}^{-1}$. PSZ2 G165.41+25.93 is also below their mass limit, whereas in our richness analysis, it has $\sigma_{\mathrm{R}}=1.8$, which is just above our validation limit of $\sigma_{\mathrm{R}}=1.5$.

We also compared our results with Zaznobin et al. (2019), who reported 38 spectroscopic redshifts for PSZ2 candidates. We find 20 matches between this catalog and the LP15 sample. We find discrepancies in only one case, PSZ2 G202.61-26.26. The authors reported three spectroscopic redshifts at $z_{\mathrm{spec}}=$ 0.533 while we find a galaxy overdensity at $z_{\text {phot }}=0.23$, but farther than $5^{\prime}$ away from the Planck center. This is therefore not linked to the SZ emission.

In order to study the galactic disturbance on the SZ Planck detection, we computed the number of nondetections as a function of the $857 \mathrm{GHz}$ signal in the Planck map. This map could be used as a tracer of thermal dust emission (Planck Collaboration XI 2014). The signal was computed as the mean value within a region of $0.5^{\circ}$ radius around the nominal pointing in the PSZ2 catalog. Figure 14 represents the ratio between the unconfirmed and the total number of sources for the PSZ2-North sample as a

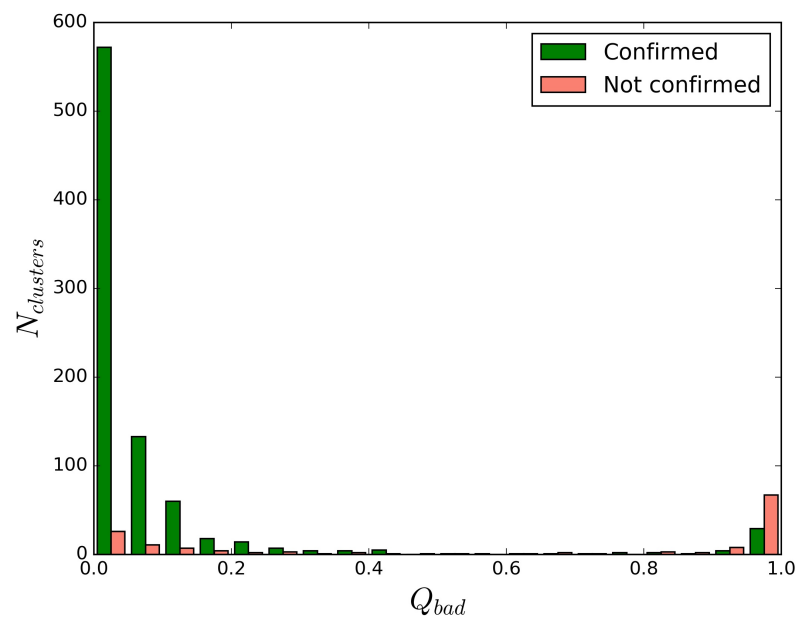

Fig. 15. Number of cluster candidates vs. the neural network quality flag value for the PSZ2-North sample. Confirmed candidates are represented in green, and still unconfirmed clusters are shown in red. The bin size is 0.05 .

function of the $857 \mathrm{GHz}$ signal in bins of $1 \mathrm{MJy} \mathrm{sr}^{-1}$. This figure shows a clear correlation between these two magnitudes. Below $7 \mathrm{MJy} \mathrm{sr}^{-1}$, the ratio of unconfirmed sources is lower than $20 \%$. However, in zones with high dust emission (mainly places in the galactic plane), the false SZ clusters can exceed 60-70\%.

Figure 15 shows the number of cluster candidates versus the neural network quality flag value for the PSZ2-North sample $\left(Q_{\text {bad }}\right)$. This value was defined in Aghanim et al. (2015). It is an indicator of how reliable an SZ source is confirmed as a real galaxy cluster. Candidates with values of $Q_{\text {bad }}>0.6$ are considered low-reliability sources. In the PSZ2-North sample, we observe that the vast majority $(>93 \%)$ of the clusters with $Q_{\text {bad }}<0.6$ are actual clusters, while fewer than $33 \%$ with $Q_{\text {bad }}>0.6$ are confirmed.

We also compared the full validation results with Khatri (2016), who published a validation method for the PSZ2 catalog based on combined $\mathrm{CO}$ and $y$-distortion maps. He classified the sources into five different groups depending on the value of his estimator: $M O C, p M O C, C L G, p C L G$, and IND. The signal of the sources classified as $M O C$ and $p M O C$ was considered to come from molecular clouds, and $C L G$ and $p C L G$ signals come from galaxy clusters. IND is indeterminable. We find that $95.2 \%$ of the IND and $94.6 \%$ of the $C L G+p C L G$ correspond to actual validated clusters. On the other hand, $64.7 \%$ of the $M O C+p M O C$ are also validated clusters. We expected that sources with this classification have a lower validation rate, but this is not the case. A possible explanation for these results is that the threshold used by Khatri (2016) to distinguish between molecular clouds and clusters was shifted toward high values of $\Delta\left(\Sigma \chi^{2}\right)_{\mathrm{CO}-y}$. To illustrate this, for the 59 sources that the author classified as pMOC, $48(81.3 \%)$ are actual clusters. We will fully study this in a future publication, where we will discuss the cluster properties, velocity dispersion, masses, and their relations with the SZ signal.

\section{Conclusions}

We have presented the final results of the observational program LP15 that we began to describe in Paper I. We reported here the second year of observations that were carried out using INT, TNG, and GTC at the ORM Observatory as part of the optical 
follow-up confirmation and characterization program of Planck SZ sources in the northern hemisphere.

During the second year of observations, 78 PSZ2 sources with no known optical counterpart have been observed. A robust confirmation criterion based on velocity dispersion, when available, and richness estimations enabled us to confirm 40 candidates, for which we provided 18 spectroscopic and 22 photometric redshifts.

We updated the information on 42 sources that have been validated in the original PSZ2 catalog but lacked a redshift estimate. We provided a spectroscopic redshift for 20 of them and a photometric redshift for 20 sources. We also studied the richness and applied the same criteria as for the candidates in order to check the associations with the SZ signal. We discovered that three previously confirmed counterparts were not present in the optical range studied here.

At the end of the whole observational program LP15, we were able to confirm 81 new cluster candidates, with a median redshift of 0.29 . The mean redshift of the catalog is 0.23 . Our main contribution appears in the redshift interval $0.4<z<0.7$, where our confirmations correspond to $20 \%$ of the total clusters that are confirmed in the PSZ2 in this range. The purity of the catalog is increased from $76.7 \%$ to $86.2 \%$.

Finally, we found a clear correlation between the number of unconfirmed sources and galactic thermal dust emission. This correlation suggests that the PSZ2 catalog contains spurious detections. Some of these false detections have been discussed here. In particular, we find that more than $50 \%$ of the sources with a mean signal in the $857 \mathrm{GHz}$ maps greater than $7 \mathrm{MJy} \mathrm{sr}^{-1}$ remain unconfirmed after this work.

Acknowledgements. This article is based on observations made with the Gran Telescopio Canarias operated by the Instituto de Astrofisica de Canarias, the Isaac Newton Telescope, and the William Herschel Telescope operated by the Isaac Newton Group of Telescopes, and the Italian Telescopio Nazionale Galileo operated by the Fundacion Galileo Galilei of the INAF (Istituto Nazionale di Astrofisica). All these facilities are located at the Spanish Roque de los Muchachos Observatory of the Instituto de Astrofisica de Canarias on the island of La Palma. This research has been carried out with telescope time awarded for the program 128-MULTIPLE-16/15B. During our analysis, we also used the following databases: the SZ-Cluster Database operated by the Integrated Data and Operation Center (IDOC) at the IAS under contract with CNES and CNRS and the Sloan Digital Sky Survey (SDSS) DR14 database. Funding for the SDSS has been provided by the Alfred P. Sloan Foundation, the Participating Institutions, the National Aeronautics and Space Administration, the National Science Foundation, the US Department of Energy, the Japanese Monbukagakusho, and the Max Planck Society. This work has been partially funded by the Spanish Ministry of Economy and Competitiveness (MINECO) under the projects ESP2013-48362C2-1-P, AYA2014-60438-P and AYA2017-84185-P. AS and RB acknowledge financial support from the Spanish Ministry of Economy and Competitiveness (MINECO) under the Severo Ochoa Programs SEV-2011-0187 and SEV-20150548. HL is funded by PUT1627 grant from the Estonian Research Council and by the European Structural Funds grant for the Centre of Excellence "Dark Matter in (Astro)particle Physics and Cosmology" TK133. Some of the results in this paper have been derived using the HEALPIX (Górski et al. 2005) package.

\section{References}

Abell, G. O., Corwin, Jr., H. G., \& Olowin, R. P. 1989, ApJS, 70, 1 Aghanim, N., Hurier, G., Diego, J. M., et al. 2015, A\&A, 580, A138 Allen, S. W., Evrard, A. E., \& Mantz, A. B. 2011, ARA\&A, 49, 409 Amodeo, S., Mei, S., Stanford, S. A., et al. 2018, ApJ, 853, 36 Barrena, R., Streblyanska, A., Ferragamo, A., et al. 2018, A\&A, 616, A42 Bertin, E., \& Arnouts, S. 1996, A\&AS, 117, 393

Bleem, L. E., Stalder, B., de Haan, T., et al. 2015, ApJS, 216, 27

Boada, S., Hughes, J. P., Menanteau, F., et al. 2019, ApJ, 871, 188

Buddendiek, A., Schrabback, T., Greer, C. H., et al. 2015, MNRAS, 450, 4248

Burenin, R. A. 2017, Astron. Lett., 43, 507

Burenin, R. A., Bikmaev, I. F., Khamitov, I. M., et al. 2018, Astron. Lett., 44, 297

Chambers, K. C., Magnier, E. A., Metcalfe, N., et al. 2016, ArXiv e-prints [arXiv:1612.05560]

Cutri, R. M., Wright, E. L., Conrow, T., et al. 2013, Explanatory Supplement to the AllWISE Data Release Products, Tech. rep.

De Propris, R., Couch, W. J., Colless, M., et al. 2002, MNRAS, 329, 87

Gladders, M. D., \& Yee, H. K. C. 2000, AJ, 120, 2148

Górski, K. M., Hivon, E., Banday, A. J., et al. 2005, ApJ, 622, 759

Hambly, N. C., MacGillivray, H. T., Read, M. A., et al. 2001, MNRAS, 326, 1279

Hasselfield, M., Hilton, M., Marriage, T. A., et al. 2013, JCAP, 7, 008

Khatri, R. 2016, A\&A, 592, A48

Komatsu, E., Smith, K. M., Dunkley, J., et al. 2011, ApJS, 192, 18

Monet, D. G., Levine, S. E., Canzian, B., et al. 2003, AJ, 125, 984

Munari, E., Biviano, A., Borgani, S., Murante, G., \& Fabjan, D. 2013, MNRAS, 430, 2638

Perrott, Y. C., Olamaie, M., Rumsey, C., et al. 2015, A\&A, 580, A95

Piffaretti, R., Arnaud, M., Pratt, G. W., Pointecouteau, E., \& Melin, J.-B. 2011, A\&A, 534, A109

Planck Collaboration I. 2014, A\&A, 571, A1

Planck Collaboration XI. 2014, A\&A, 571, A11

Planck Collaboration XXIX. 2014, A\&A, 571, A29

Planck Collaboration XXII. 2016, A\&A, 594, A22

Planck Collaboration XXIV. 2016, A\&A, 594, A24

Planck Collaboration XXVII. 2016, A\&A, 594, A27

Planck Collaboration VI. 2018, A\&A, submitted [arXiv:1807.06209]

Planck Collaboration Int. I. 2012, A\&A, 543, A102

Planck Collaboration Int. XXVI. 2015, A\&A, 582, A29

Planck Collaboration Int. XXXVI. 2016, A\&A, 586, A139

Predehl, P., Andritschke, R., Böhringer, H., et al. 2010, Proc. SPIE, 7732, $77320 \mathrm{U}$

Rykoff, E. S., Rozo, E., Busha, M. T., et al. 2014, ApJ, 785, 104

Skrutskie, M. F., Cutri, R. M., Stiening, R., et al. 2006, AJ, 131, 1163

Streblyanska, A., Barrena, R., Rubiño-Martín, J. A., et al. 2018, A\&A, 617, A71

Streblyanska, A., Aguado-Barahona, A. R., Ferragamo, A., et al. 2019, A\&A, 628, A13

Sunyaev, R. A., \& Zeldovich, Y. B. 1972, Comments Astrophys. Space Phys., 4, 173

van der Burg, R. F. J., Aussel, H., Pratt, G. W., et al. 2016, A\&A, 587, A23

Voges, W., Aschenbach, B., Boller, T., et al. 1999, VizieR Online Data Catalog: IX/010

Voges, W., Aschenbach, B., Boller, T., et al. 2000, VizieR Online Data Catalog: IX/029

Wen, Z. L., \& Han, J. L. 2018, MNRAS, 481, 4158

Wen, Z. L., Han, J. L., \& Liu, F. S. 2012, ApJS, 199, 34

Wright, E. L., Eisenhardt, P. R. M., Mainzer, A. K., et al. 2010, AJ, 140, 1868

York, D. G., Adelman, J., Anderson, Jr., J. E., et al. 2000, AJ, 120, 1579

Zaznobin, I. A., Burenin, R. A., Bikmaev, I. F., et al. 2019, Astron. Lett., 45, 49

Zohren, H., Schrabback, T., \& van der Burg, R. F. J. 2019, MNRAS, 488, 1519

Zwicky, F. 1933, Helvet. Phys. Acta, 6, 110 
A\&A 631, A148 (2019)

\section{Appendix A: Additional tables}

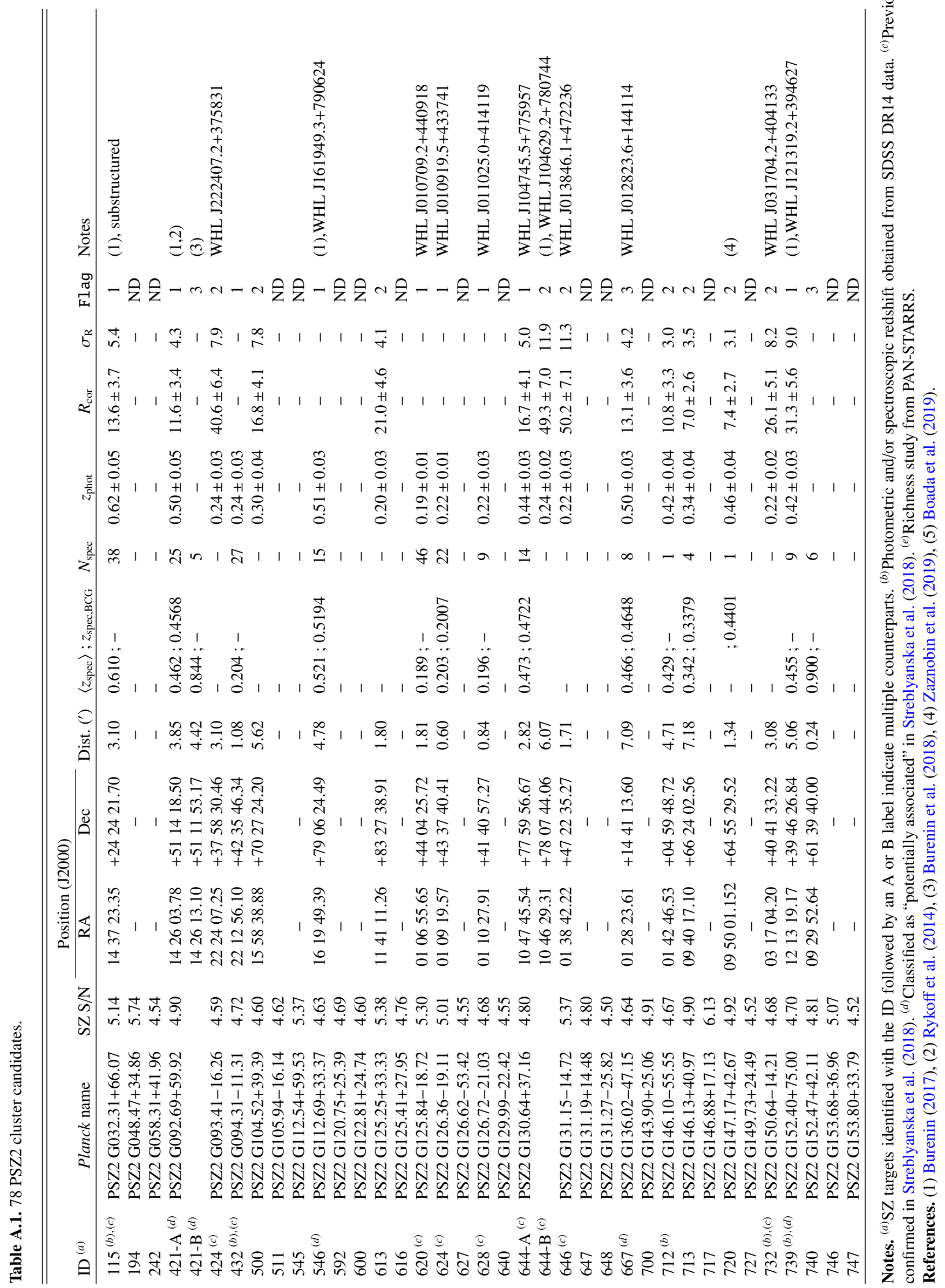


A. Aguado-Barahona et al.: Optical validation of Planck PSZ2. II.

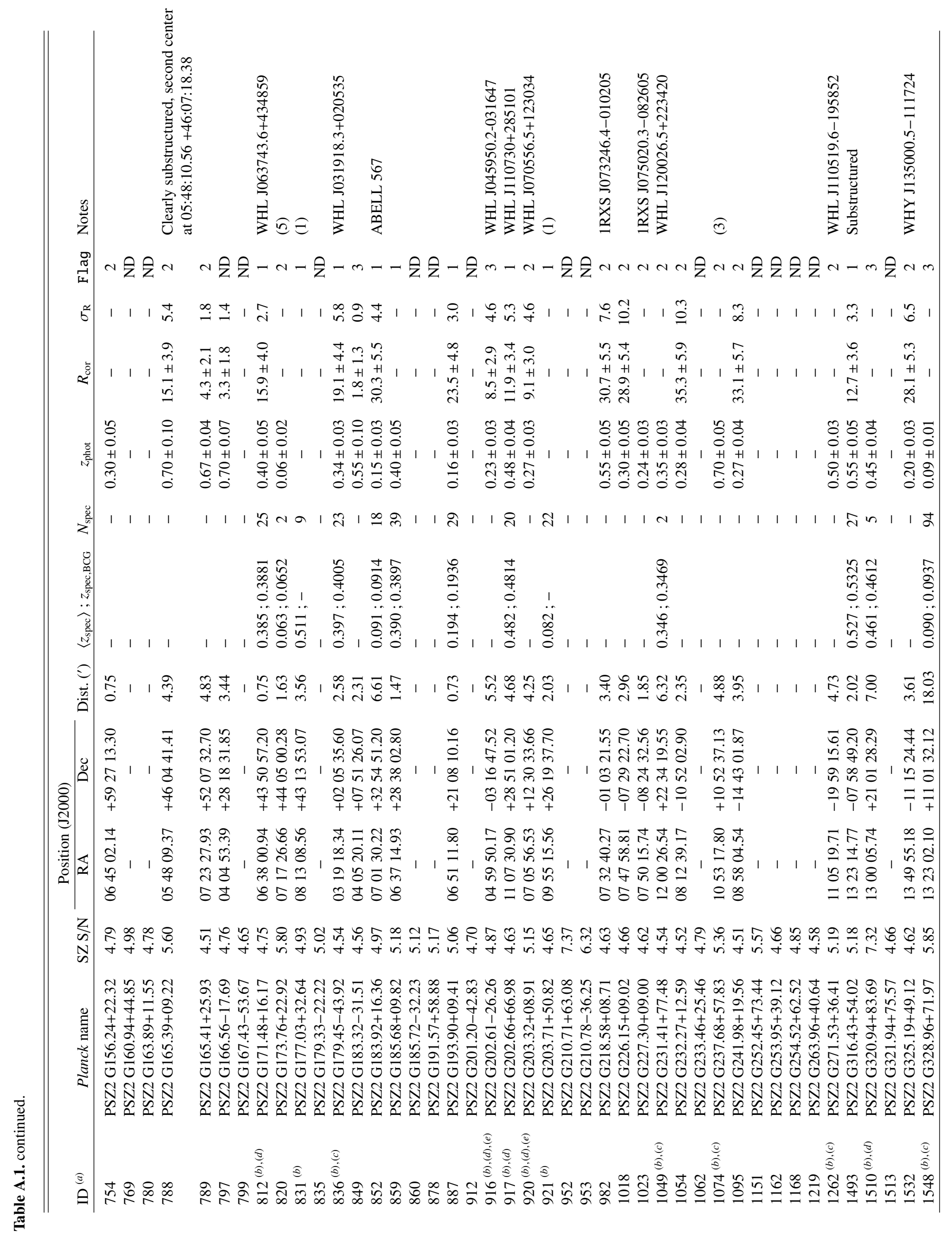




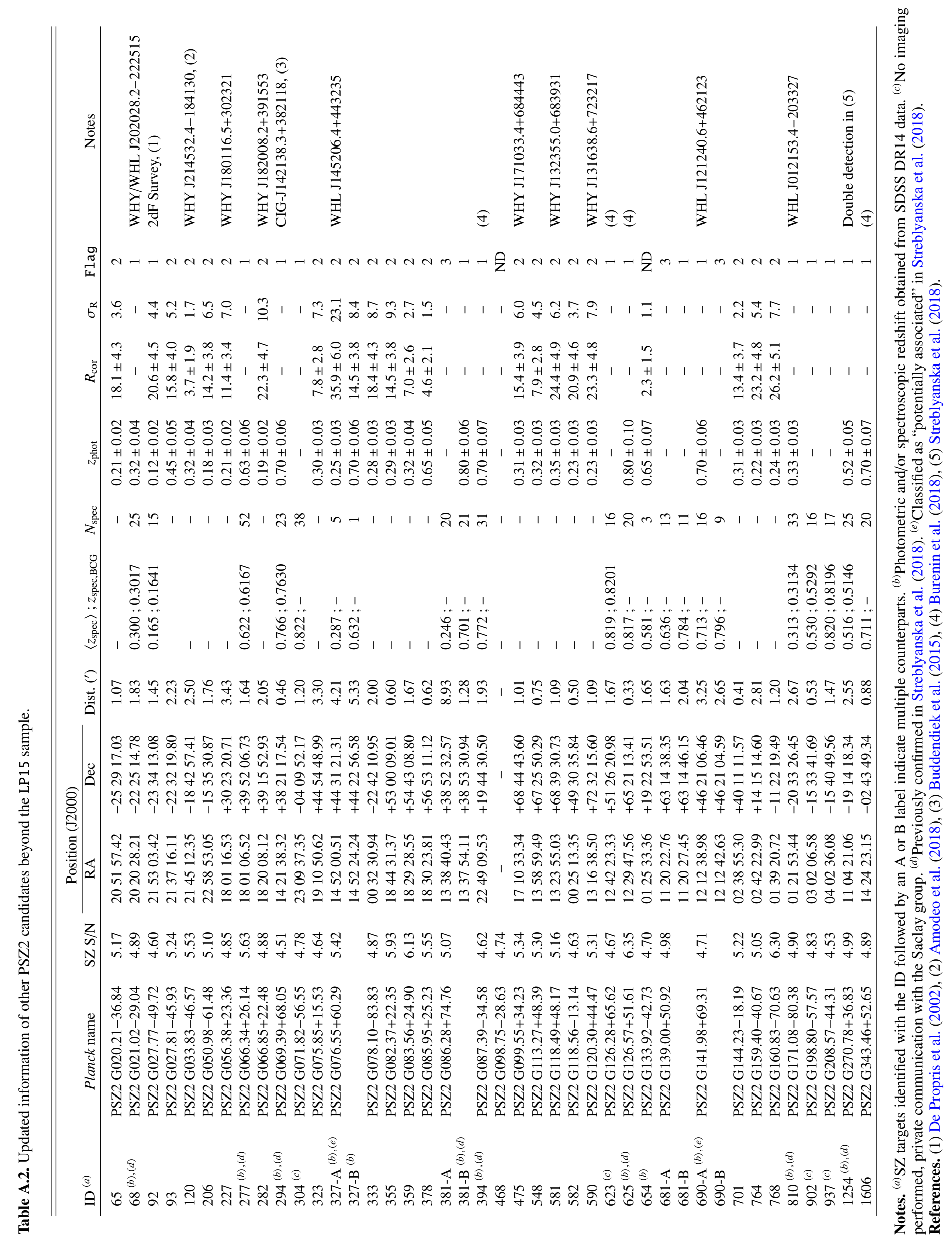

